\title{
Covid 19 in sub-Saharan Africa: Is it the calm before the storm?
}

\author{
FLORY MUANDA ${ }^{1}$, Maëlle Dandjinou ${ }^{2}$, and Hugues Sampasa- Kanyinga ${ }^{3}$ \\ ${ }^{1}$ Western University Schulich School of Medicine and Dentistry \\ ${ }^{2}$ University of Montreal Faculty of Pharmacy \\ ${ }^{3}$ Affiliation not available
}

May 5, 2020

\begin{abstract}
We proposed several hypotheses to explain the low rate of Covid19 in Sub-Sahara Africa (SSA). The small number of people tested for Covid19, a younger population, higher immunity to covid19, and seasonality emerged as potential factors influencing the Covid 19 rate in SSA. Rigorous responses to covid19 to flatten the curve are urgently needed and will include (1) a substantial increase of Covid19 testing and the use of cellphone location to trace contact, (2) complete lockdowns with social distancing measures followed by an assessment of the impact of those interventions to flatten the curve, (3) the use of prior experience with Ebola outbreak to increase awareness about the Covid-19 and its fatal complications, (4) warnings about potential side effects associated with the use of chloroquine/hydroxychloroquine with azithromycin and (5) the maintenance of essential health services
\end{abstract}

Since the beginning of the coronavirus disease (Covid-19) Pandemic in Wuhan (China), Sub-Saharan Africa (SSA) seems to be the least affected region worldwide. As of April 21, 2020, the number of Covid-19 cases per 1 million persons in the three most populated countries, including Nigeria, Ethiopia, and the Democratic Republic of Congo (DRC) was 3.8, 1.0, and 4.0, respectively. ${ }^{1,2}$ These rates are neatly lower than the average rate reported worldwide (329.9 cases per 1 million persons on April 21, 2020). ${ }^{1}$

We proposed several hypotheses to explain this discrepancy with the other parts of the world, such as Europe and the United States of America (USA); (1) there is a low number of people tested for Covid-19 in SSA. For example, in the DRC, 806 tests were performed as of April 21, 2020, approximately less than two tests per 100000 persons every day, which underestimated the true number of cases. Conversely, South Africa had the higher number of tests done in Africa (approximately 126,937) as well as the higher number of Covid-19 cases, with 3465 cases equivalent to 58.4 per 1 million persons. ${ }^{2}$ (2) African population may be more immune to Covid-19 compared to the Western or American population. Recent evidence suggest that countries with mandatory policies to vaccinate against tuberculosis, including Japan and South Korea, reported fewer number of confirmed cases and deaths from Covid- $19 .{ }^{3}$ Although vaccination against tuberculosis is mandatory in SSA, it remained unclear whether these results can apply to the African population. (3) African population is younger compared to the Western population. The median population age is 43.1 in Europe, 38.4 in China, but only 19.7 in Africa. Current literature suggests that older people are at higher risk of Covid-19 and related complications. (4) It might be a matter of time. SSA might be at an early stage of the Covid-19 pandemic. The first case of Covid-19 in SSA was reported in Nigeria on February $27^{\text {th }}, 2020$. One month and a half later (i.e., April 21, 2020), this number increased to over 24,000 confirmed cases of coronavirus across Africa. ${ }^{2}$ (5) The seasonality might play an important role in the occurrence of Covid-19 infections. Prediction research suggested that regions with temperate warm and cold climates followed by regions with arid climates would be the most affected, while regions with tropical climates should be less affected by this pandemic. ${ }^{4}$ 
Despite the low incidence of Covid-19, SSA has quickly adopted important measures to slow down the spread of the pandemic. As of March 30, 2020, 46 of sub-SSA's 49 sovereign states have imposed partial or full closures of their borders; 44 have closed schools, banned public gatherings, or put in place other social distancing measures; and 11 have declared a state of emergency. However, in many countries, the number of confirmed cases has increased exponentially even after the implementation of these measures, suggesting a community transmission of the virus. For example, South African citizens returning from high-risk countries have been tested and put on self-isolation or quarantine upon their return to South Africa since March $17^{\text {th }}$, 2020. Regardless of these measures, the number of Covid-19 cases have jumped from 1353 on March 31, 2020 to 3465 cases on April 21, 2020. ${ }^{2}$

The current situation in SSA calls for immediate action to slow down transmission and avoid the collapse of the healthcare system. With five intensive care beds per million people, few ventilators for millions of people, two doctors and 11 nursing/midwifery personnel per 10,000 population, SSA will not be able to face this pandemic, if the healthcare system gets overwhelmed.

In the absence of a vaccine, more rigorous strategies tailored to the local context are urgently needed to flatten the Covid-19 curve in SSA. (1) Many African countries should increase their capacity to test Covid-19 and trace contact using cellphone location as in South Korea. The widespread use of cellphones in urban cities (the most affected areas in SSA) is an opportunity to contain the spread of Covid-19. SSA should also test people at higher risk of contracting Covid-19 infections, including health care professionals, older adults as well as people living with HIV and tuberculosis.

(2) complete lockdowns with social distancing measures, frequent handwashing, and regularly disinfect hightouch surfaces are currently the most effective strategy to control the spread of this virus worldwide. Although it is difficult to achieve in SSA, those policies are vital since many lives are at stake. For example, governments, in collaboration with not-for-profit organizations, may create food banks and ensure distribution to the African population with priority to the poorer. SSA should also work closely with epidemiologists to assess whether those measures are effective at flattening the curve. A study conducted by the Imperial College Covid-19 response team predicted that 300.000 deaths would occur by the end of the year if people respected an intense social distancing. Conversely, if no intervention was implemented in Africa, this number would increase to 3.5 million deaths by the end of the year.

(3) SSA should use its prior experience with Ebola outbreak to increase awareness of African population about the Covid-19 and its fatal complications. Each African government may also share with their fellow citizens results from prediction models using their data to increase awareness and save millions of lives.

(4) Physicians who prescribed chloroquine/hydroxychloroquine with azithromycin for the treatment of Covid19 should be aware of potential side effects even during a short course of treatment. As we await stronger evidence to support the role of chloroquine/hydroxychloroquine with azithromycin in the treatment of Covid-19, many countries, including those in SSA, have adopted these combinations for the management of the disease. As such, clinicians prescribing chloroquine/hydroxychloroquine should be cognizant of the following side effects, including prolongation of QTc interval (especially in a patient with pre-existing cardiac disease or when used in combination with azithromycin), hypoglycemia, neuropsychiatric effect, drug-drug interaction with beta-blocker (carvedilol) or opioids (codeine, tramadol) and idiosyncratic hypersensitivity reactions (StevensJohnson syndrome). ${ }^{5}$ Although the risk is low, a close follow-up with patients to identify early signs of side effects should be mandatory, and self-medication with the combinations of chloroquine/hydroxychloroquine with azithromycin should be contraindicated.

(5) SSA should maintain essential health services (routine vaccination; reproductive health services including care during pregnancy and childbirth; care of young infants and older adults; management of mental health conditions as well as noncommunicable diseases and infectious diseases like HIV, malaria, and tuberculosis ...) during the Covid-19 pandemic. Failure to do so would increase non-Covid-19 hospitalizations, which would have a detrimental effect on flattening the curve.

(6) SSA should be part of current research on Covid-19 to ensure that future treatment and vaccine are also 
effective in an African population. Of the 745 studies reported on the website clinicaltrials.gov, only three studies $(0.4 \%)$ will be conducted in SSA. However, some African governments encourage local initiative of research on Covid-19 using improved traditional medicine (i.e Manacovid, Covid-Organics, and Apivirine). To date, no randomized controlled trials have been conducted to test their efficacy.

In conclusion, the Covid-19 rate is low but on the rise in SSA. In collaboration with traditional partners and not-for-profit organizations, a global response with rigorous strategies tailored to the local context is urgently needed to flatten the Covid-19 curve and save millions of lives in SSA.

\section{Conflict of Interest Disclosures}

All the authors declared no conflict of interests

References

1. World Health Organisation.Explore the data. Geneva.2020. (https://covid19.who.int/explorer.)

2. Africa Centres for Disease Control and Prevention. COVID-19 dashboard. (https://africacdc.org/covid19/.)

3. Gursel M, Gursel I. Is Global BCG Vaccination Coverage Relevant To The Progression Of SARS-CoV-2 Pandemic? Med Hypotheses 2020;109707.

4. Araujo MB, Naimi B. Spread of SARS-CoV-2 Coronavirus likely to be constrained by climate. medRxiv;2020.

5. Juurlink DN. Safety considerations with chloroquine, hydroxychloroquine and azithromycin in the management of SARS-CoV-2 infection. CMAJ;2020. 